\title{
EXPERIMENTAL VALIDATION OF NUCLEAR REACTION DATA FOR INVENTORY SIMULATIONS ON MOLYBDENUM
}

\author{
M.R. Gilbert, L.W. Packer and T. Stainer \\ Culham Centre for Fusion Energy, UKAEA, Culham Science centre, \\ Abingdon, Oxfordshire, UK, OX14 3DB \\ mark.gilbert@ukaea.uk, lee.packer@ukaea.uk, thomas.stainer@ukaea.uk
}

\begin{abstract}
Molybdenum is being considered as a potential material for future nuclear fusion experiments and power plants. It has good thermo-mechanical properties and can be easily fabricated, making it attractive as an alternative first wall material to tungsten, which is the current leading candidate. Unfortunately, according to simulations, fusion-neutron irradiated Mo may become too activated during reactor operation to maintain the objective of fusion of avoiding the generation of long-term, higher-level radioactive waste.

However, these simulated predictions rely heavily on having high-quality, accurate nuclear reaction data. For example, reliable simulations of the radiological response of isotopically-tailored Mo would be required by reactor designers and eventually nuclear regulators to assess if it is a viable low-activation fusion solution (in comparison to pure Mo). In recent years, UKAEA has developed benchmarks to test and validate the FISPACT-II inventory code and the input international nuclear data libraries against experimental measurements. This paper presents nuclear code prediction comparisons to new data acquired from $\gamma$-spectroscopy measurements of Mo irradiated in the ASP $14 \mathrm{MeV}$ facility at AWE in the UK.

Results demonstrate that FISPACT-II predictions for Mo are remarkably accurate; particularly for activity generated from the shorter-lived radionuclides explored by these laboratory experiments, such as ${ }^{91} \mathrm{Mo}$ and ${ }^{97} \mathrm{Nb}$, etc., and their metastable isomeric states.
\end{abstract}

KEYWORDS: Molybdenum, fusion irradiation, experimental validation, nuclear data

\section{INTRODUCTION: MOLYBDENUM AS A FUSION MATERIAL}

Molybdenum is often proposed as an alternative material for the high neutron flux and high thermal load regions - the plasma-facing components (PFCs) - of nuclear fusion devices [1,2]. While tungsten is the main candidate [3,4], difficulties with fabrication and issues surrounding irradiationinduced embrittlement [3] lead to continued interest in alternatives, such as Mo. Molybdenum exhibits similarly low sputtering yields [1] and equivalent thermal-mechanical properties to tungsten, while being potentially easier to fabricate into fusion components [5].

However, a key drawback for Mo as a fusion material concerns its induced radioactivity under neutron irradiation. The predicted $\gamma$ dose rate measured in Sieverts per hour (significant in determining whether a material can be manipulated by remote-handling equipment) from Mo at timescales of 
50-100 years beyond fusion reactor end-of-life (EOL) are predicted to be several orders of magnitude higher for molybdenum compared to either tungsten or iron (the predominant element in structural components). For example, after exposure to first wall armour conditions in DEMO, simulations suggest that dose rates in Mo will still be of the order of $1 \mathrm{~Sv} / \mathrm{hr}$ after 100 years of decay, compared to around $10 \mu \mathrm{Sv} / \mathrm{hr}$ from either Fe or $\mathrm{W}$ [2]. This makes it difficult to justify Mo for fusion applications where the goal is to avoid the generation of higher-level waste requiring long-term deep geological disposal [6].

On the other hand, calculations in [2] demonstrated that only some of the stable Mo isotopes have pathways that result in particularly high dose rates via activation. Several isotopes $-{ }^{96} \mathrm{Mo}$, ${ }^{97} \mathrm{Mo},{ }^{98} \mathrm{Mo}$ and ${ }^{100} \mathrm{Mo}$ - produce relatively low doses at long timescales compared to natural Mo. This offers a potential solution that could make Mo more viable for fusion applications - instead of natural Mo, use Mo with an artificially adjusted (tailored) distribution of stable isotopes, preferably dominated by ${ }^{96} \mathrm{Mo}$ and ${ }^{97} \mathrm{Mo}$ [2]. There are various possible options for isotopic enrichment of Mo, including via cascades in gas centrifuges [7], or via a free electron laser of $\mathrm{CO}_{2}$ [8], although it is recognised that the (likely) high cost of using several tonnes of isotopically enriched Mo as a first wall material in a fusion reactor would have to be balanced against the potential benefit - e.g. of having reduced costs for waste disposal.

Alternatively, Mo with a modified isotope distribution could be useful to reduce the activation of fusion alloys, such as the ferritic-martensitic steels needed for in-vessel reactor components. For example, previous work [9] suggests that using tailored Mo in EUROFER steel might be a reasonably economic solution to reduce maintenance requirements and improve thermodynamic efficiency.

Mo is also touted as having potential applications as a structural material in fuel elements to improve safety in future generations of fission power plants [10]. In this case, the barrier to realisation is the very high (thermal) capture cross section for neutrons in natural Mo, which is obviously undesirable when trying to create a sustained nuclear reaction. Once again, isotopic tailoring to reduce the problematic isotopes (in this case mainly ${ }^{95} \mathrm{Nb}$ [10]) offers a possible solution. Excessively high neutron capture cross sections could also be a problem for fusion, although in that case, molybdenum's potential application in fusion PFCs would not be concerned with thermal neutron capture as such locations in a reactor are overwhelmingly dominated by fast, $14 \mathrm{MeV}$ neutrons (i.e. the neutron energies generated as a result of the deuterium-tritium [DT] fusion reaction), but it could be a concern if Mo is used in significant concentrations in the tritium-breeding zone, where the loss of neutrons in a material with a high absorption cross section could impact on the TBR (tritium breeding ratio) [11].

Predictions such as those discussed in [2] rely on accurate nuclear code simulations. While the numerical techniques employed by inventory codes like FISPACT-II [12] or neutron transport simulators like MCNP are well-established and validated (see e.g. [13,14]), it is still the case that the quality and completeness of the underlying nuclear data strongly determines reliability of predictions. Of particular importance for simulating the radiological response of molybdenum (or any other material) under fusion conditions is the accuracy of the integrated reaction rates that govern the production of dominant radionuclides. This paper describes recent efforts at UKAEA to test and validate the nuclear data libraries used with FISPACT-II, focussing on important nuclear reactions for Mo. 


\section{ASP EXPERIMENTS}

In an effort to improve the quality of experimental reaction cross section data for fusion-relevant materials at fusion-relevant neutron energies, UKAEA undertook a series of $14 \mathrm{MeV}$ neutron irradiation experiments at the experimental facility known as 'ASP', which is hosted at AWE Aldermaston in the UK. The aim of the experimental campaigns was to gain additional cross section data-points at $14 \mathrm{MeV}$ for reactions where data was deficient - this is true for many important reactions for fusion materials $[15,16]$ - and thus to aid the evaluators working on the next generation of nuclear data libraries by providing more information with which to fit the theoretical models that generate continuous cross section curves.

The experimental approach involved a deuteron beam onto a tritium-loaded target to produce a source of $14 \mathrm{MeV}$ neutrons, which was then used to irradiate a wide variety of material foils. After irradiation the foils were transferred (rapidly) to a high-purity germanium (HPGe) $\gamma$-detector and the full time evolution in the energy-count profile of $\gamma$ emissions from the activated material was recorded. Full details of the experimental approach are given in [17,18]. So far, more than 300 separate experiments have been performed by UKAEA, generating more than 10000 separate $\gamma$ spectra (the integral spectrum is recorded at multiple time intervals for each experiment to enable analysis - see below), and previous efforts [19] to process such large data sets in a rigorous, consistent and automatic manner are still ongoing [20]. In the present work, we analyse eight experiments involving samples of Mo.

Figure 1 shows the typical raw data obtained from the ASP experiments, along with an example of the analysis approach. The data shown corresponds to experiment 82 in UKAEA's campaign. The full, time-integrated $\gamma$-spectrum (figure 1a) recorded for 15 minutes after irradiating a foil triplet of Mo-Fe-Al shows various peaks or lines at characteristic $\gamma$-energies for different radionuclides present in the foils after a 5-minute irradiation (both the irradiation time and measurement time varied between experiments, but was accurately recorded). The main lines are highlighted in the figure, including background peaks and those associated with radionuclides produced during irradiation of Mo, Fe and Al.

Iron $(\mathrm{Fe})$ and aluminium ( $\mathrm{Al})$ foils were included in the experiments to enable accurate measurement of the neutron fluence received at the sample location during irradiation, as described in [19]. In the present work, the major peaks from ${ }^{27} \mathrm{Mg}$ and ${ }^{24} \mathrm{Na}$ due to aluminium activation, along with ${ }^{56} \mathrm{Mn}$ produced in the Fe foil were used to produce an average estimate of the neutron flux for each experiment. Note that for experiment 81 , a transfer issue meant that the counts from the aluminium foil were not properly recorded and so the flux estimate is based solely on the ${ }^{56} \mathrm{Mn}$ peaks in this case. For the eight experiments considered here, the typical estimated neutron flux is around $5 \times 10^{8} \mathrm{n} \mathrm{cm}^{-2} \mathrm{~s}^{-1}$.

The estimated fluxes were used to both evaluate the experimental $E$ activity of radionuclides in the molybdenum foils and also as input to FISPACT-II inventory simulations to obtain a calculated $C$ activity. For the experimental value, the first stage is to extract the detector counts for each detectable $\gamma$-peak of the $\gamma$-emitting radionuclides produced during the irradiation of Mo at ASP. Peaks associated with five different radionuclides were identified in the present work; these are listed in table 1, together with their half-lives, and main production pathways according to FISPACT-II simulations with TENDL-2017 for the irradiation time and flux-estimate associated 


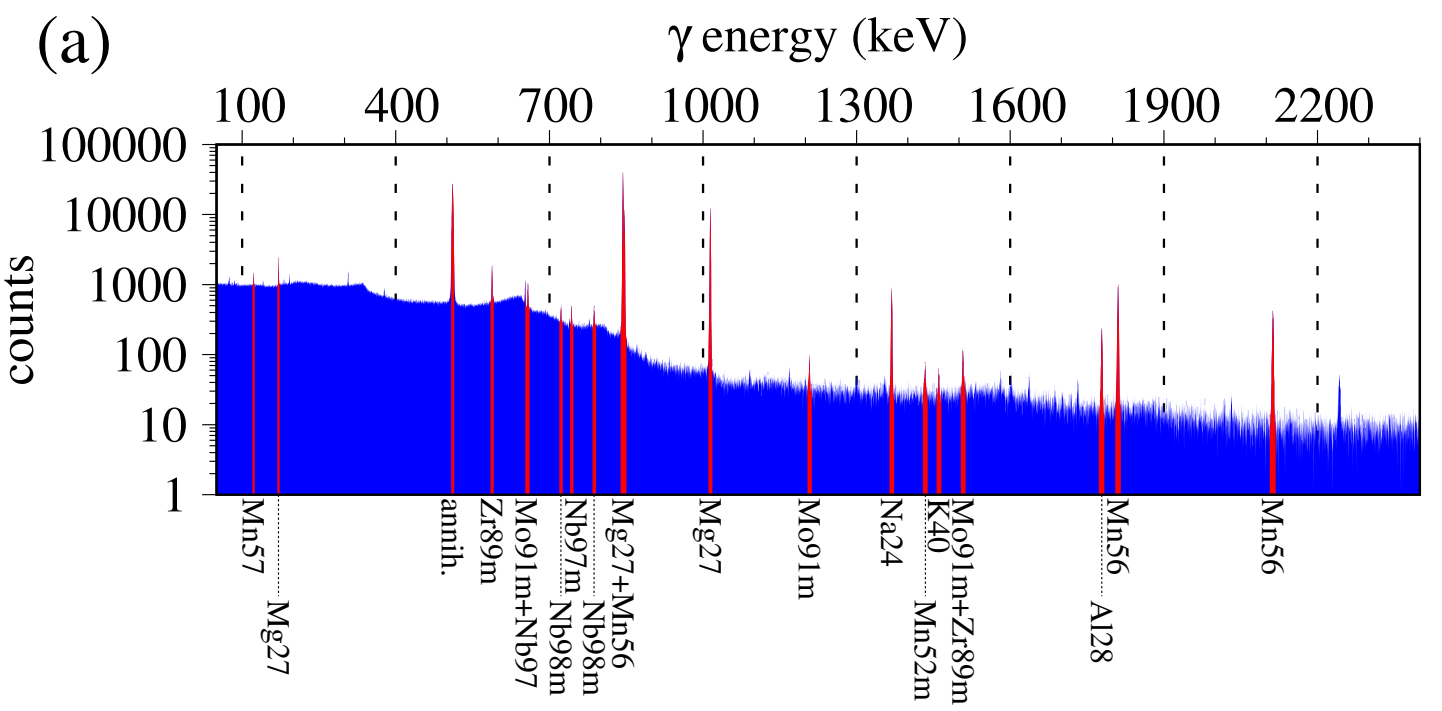

(b)
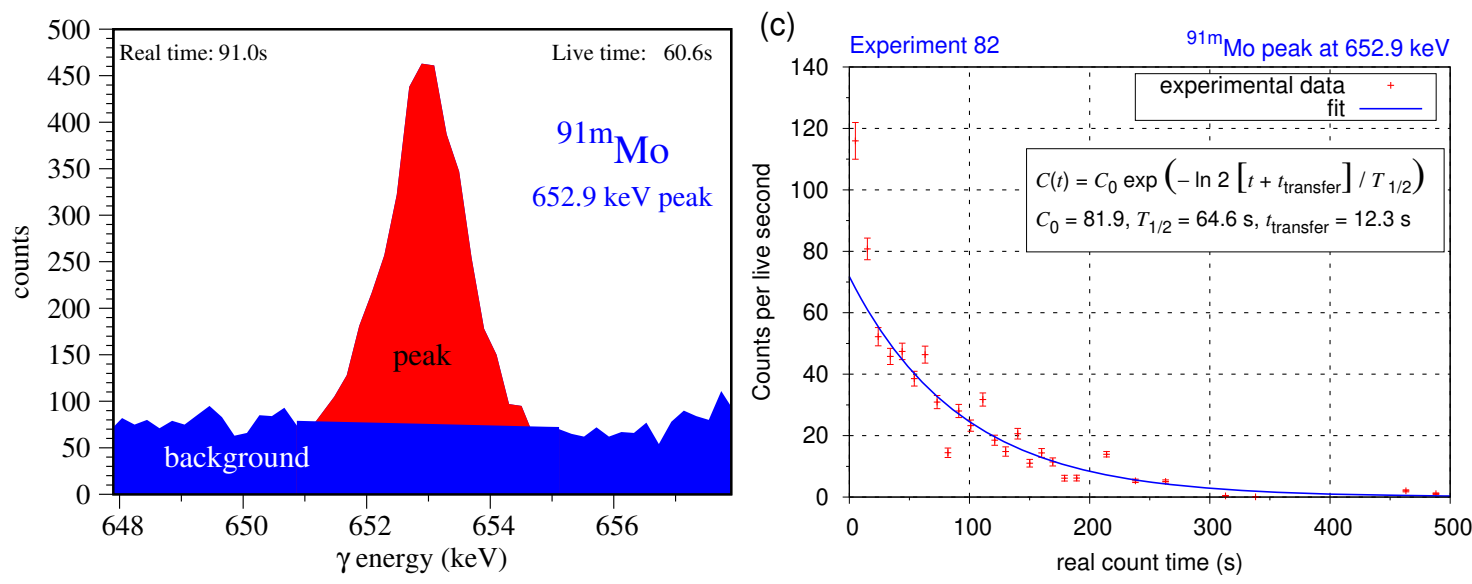

Figure 1: Experiment $82 \gamma$ spectroscopy. (a): final (end of $\sim 15-$ min. acquisition) integrated $\gamma$ spectrum. (b): $652.9 \mathrm{keV}$ peak associated with ${ }^{91 m}$ Mo after $91 \mathrm{~s}$, showing the peak area after subtraction of the background. (c): counts as a function of time for this peak and the curve fitted to the data (see main text for details). $C_{0}$ is the dependent (free) variable. $t_{\text {transfer }}$ is the time delay between the end of the irradiation and the start of the $\gamma$-data acquisition - i.e. the transfer time.

with experiment 82 .

Figure $1 \mathrm{~b}$ demonstrates the process by which the background counts (from Compton scattering) are subtracted, using linear interpolation between the average counts recorded in a range of channels on either side of a peak [19], to give the counts associated with the decaying nuclide - in this case ${ }^{91 m} \mathrm{Mo}$ at $653 \mathrm{keV}$. The fit, using the Levenberg-Marquardt algorithm (LMA - a damped leastsquares method), to the resulting evolution in counts-per-live-second as a function of (real) time for a peak (e.g. as in figure $1 \mathrm{c}$ for the ${ }^{91 \mathrm{~m}}$ Mo peak) then gives the experimentally predicted count rate at the end of irradiation $C_{0}$, which in turn is used to calculate $A_{0}$ via

$$
A_{0}=\frac{C_{0}}{D^{\mathrm{eff}}\left(E_{p}\right) I_{p}}
$$


where $D^{\text {eff }}(E)$ is the detector efficiency energy $E$, which for the HPGe detector used in the present work is described by a function fit to neutron transport simulations performed on a model of the detector (e.g. as described in [20]). $E_{p}$ and $I_{p}$ are the energy and intensity of peak $p$, both taken from decay data files used by FISPACT-II [12].

Meanwhile, the calculated estimate of $A_{0}$ is taken directly from a FISPACT-II calculation with the TENDL-2017 data library. A sample of Mo, with the same mass as used in the experiments was irradiated for the same time as recorded for the experiment at the estimated flux under the calculated neutron spectrum. FISPACT-II automatically outputs the individual radionuclide contributions to the total sample activity and end-of-irradiation values can be easily extracted for the required nuclides.

Figure 2 shows all of the $C / E$ values obtained from the eight Mo experiments. Table 1 lists the identifiable $\gamma$-peaks for each of the five nuclides considered (in figure $2 C / E$ results for ${ }^{97} \mathrm{Nb}$ and ${ }^{97 m} \mathrm{Nb}$ are plotted in the same pane). The error estimates, shown as error bars in the figure, include the standard error (i.e. the deviation of the flux values from the mean) of the flux estimate, the fractional error calculated by FISPACT-II and associated with the uncertainty data in TENDL, and the standard statistical count $\sqrt{N} / N$ uncertainty commonly applied to detector results. Work is ongoing to refine these uncertainties and account for additional (systematic) errors.

Note in figure 2 that not every experiment has produced a $C / E$ value for every possible peak considered. Peaks with total background-corrected counts of less than 400 during the entire measurement time were deemed to be of low statistical quality and were omitted. Also shown for each pane is the weighted average $C / E$ value, where the weights correspond to the inverse of the variance (square of the error) for each point. The standard deviation from these weighted averages is shown by the grey band in each plot.

The results for ${ }^{89 m} \mathrm{Zr}$ are particularly good, with $C / E$ near to one for almost all experiments. Only experiment 134 produces a discrepant result for this radionuclide - as it does for other nuclides considered. The irradiation time in this experiment was longer than normal (1 hour compared to the usual 5 minutes) and the detector measurements were extended to an over-night count. The increased irradiation time, in particular, appears to have caused the peaks from the radionuclides of interest to have more background noise (potentially from $\mathrm{Fe} \& \mathrm{Al}$ ) and thus lower overall counts than expected, leading to an underestimate from the experiment and high $C / E$ results.

For ${ }^{91 m} \mathrm{Mo}$, only the low-energy peak at $653 \mathrm{keV}$ produces consistently good $C / E$ values, which is unexpected as the $C$ values for a given experiment are identical for all peaks. $653 \mathrm{keV}$ is the highest intensity peak $\left(I_{p} \approx 0.5\right)$. The other, higher-energy peaks for this radionuclide have lower intensities and thus produce smaller count-rates in the experiments - the $653 \mathrm{keV}$ peak integral typically comprised 2000-4000 counts for this nuclide, while the other two produced a maximum of around 700 counts each (some counts were even below the 400 threshold discussed above). This may explain the discrepancy observed in figure 2 .

Apart from experiment 134, for the reasons outlined above, $C / E$ results for ${ }^{98 m} \mathrm{Nb}$ are very good and relatively consistent for the two different peaks identified in the experiments. Similarly, results for ${ }^{97} \mathrm{Nb}$ and ${ }^{97 m} \mathrm{Nb}$ (one peak each) show consistency for each experiment, but the calculated values appear to underestimate the production of these two nuclides; the weighted average $C / E$ is 
around 0.8 . The results for these nuclides need further investigation - probably involving additional experiments.

Generally, considering all of the experimental uncertainties, but particularly those associated with the flux estimation, the fact that many of the $C / E$ values are close to one (and all are less than 2) is very encouraging and demonstrates that the FISPACT-II-with-TENDL-2017 calculations produce good predictions for irradiation-induced activity in Mo in these scenarios. However, it is worth observing that none of the reaction pathways interrogated by these experiments are relevant for the long-lived radionuclides identified in the earlier calculations [2,21] as being dominant in fusionirradiated Mo at long decay times. For these nuclides, which include ${ }^{91} \mathrm{Nb},{ }^{93} \mathrm{Mo}$, and ${ }^{93 m} \mathrm{Nb}[21]$, the dominant production cross sections can have high uncertainty (e.g. the (n,np) channel responsible for the majority of ${ }^{93 m} \mathrm{Nb}$ production has a nuclear data uncertainty greater than $50 \%$ in the TENDL-2017 under $14 \mathrm{MeV}$ neutrons). This demonstrates the need for future experiments with careful design; i.e. to ensure that the important reaction pathways are explored and that the resulting benchmark is as relevant as possible for Mo in a fusion power plant.

Table 1: Information about the radionuclides measured in the ASP experiments. The FISPACT-II-calculated production pathways and their \% contributions were obtained from the simulations with the TENDL-2017 library (see the main text). Only the $\gamma$-peak energies observed in the experiments are listed for each nuclide.

\begin{tabular}{|c|c|c|c|c|}
\hline Product & $\mathbf{T}_{1 / 2}$ & Pathways & $\begin{array}{l}\text { Path \% } \\
\text { TENDL-2017 } \\
\text { (Exp. 82) }\end{array}$ & $\begin{array}{c}\text { Experimentally } \\
\text { Identifiable } \\
\gamma \text {-peaks }(\mathrm{keV})\end{array}$ \\
\hline${ }^{91 m}$ Mo & $1.08 \mathrm{~min}$. & ${ }^{92} \mathrm{Mo}(\mathrm{n}, 2 \mathrm{n}){ }^{91 m} \mathrm{Mo}$ & 100.0 & $652.9,1208.1,1508.0$ \\
\hline${ }^{98 m} \mathrm{Nb}$ & $51.30 \mathrm{~min}$. & ${ }^{98} \mathrm{Mo}(\mathrm{n}, \mathrm{p}){ }^{98 m} \mathrm{Nb}$ & 100.0 & $722.6,787.4$ \\
\hline${ }^{97} \mathrm{Nb}$ & 1.23 hours & $\begin{array}{l}{ }^{97} \mathrm{Mo}(\mathrm{n}, \mathrm{p}){ }^{97} \mathrm{Nb} \\
{ }^{98} \mathrm{Mo}(\mathrm{n}, \mathrm{np}){ }^{97} \mathrm{Nb} \\
{ }^{98} \mathrm{Mo}(\mathrm{n}, \mathrm{d}){ }^{97} \mathrm{Nb}\end{array}$ & $\begin{array}{l}81.5^{1} \\
12.1^{1} \\
6.3^{1}\end{array}$ & 657.9 \\
\hline${ }^{97 m} \mathrm{Nb}$ & $53.0 \mathrm{~s}$ & $\begin{array}{l}{ }^{97} \mathrm{Mo}(\mathrm{n}, \mathrm{p}){ }^{97 m} \mathrm{Nb} \\
{ }^{98} \mathrm{Mo}(\mathrm{n}, \mathrm{np}){ }^{97 m} \mathrm{Nb} \\
{ }^{98} \mathrm{Mo}(\mathrm{n}, \mathrm{d}){ }^{97 m} \mathrm{Nb}\end{array}$ & $\begin{array}{l}83.7 \\
2.1 \\
14.1\end{array}$ & 743.4 \\
\hline${ }^{89 m} \mathrm{Zr}$ & $4.13 \mathrm{~min}$. & ${ }^{92} \mathrm{Mo}(\mathrm{n}, \alpha)^{89 m} \mathrm{Zr}$ & 100.0 & 587.8 \\
\hline
\end{tabular}

${ }^{1}$ includes contribution from the production and isomeric transition (IT) decay of ${ }^{97 m} \mathrm{Nb}$, but note that different reactions have different probability ratios between ground- and meta-state production (i.e. the distribution of production \% values for ${ }^{97 m} \mathrm{Nb}$ are different to those of ${ }^{97} \mathrm{Nb}$ ).

\section{CONCLUSIONS}

The present experimental validation efforts for nuclear inventory code predictions of $14 \mathrm{MeV}$ neutron-induced activity in Mo have shown that the simulation methodology (FISPACT-II) and underlying nuclear data are reasonably successful. $\gamma$-spectroscopy-derived activation estimates for radionuclides produced in Mo foils irradiated at the ASP $14 \mathrm{MeV}$-neutron source in the UK have provided a wealth of new data to test code predictions against. For Mo, eight separate experiments were used to derive end-of-irradiation activities for five different radionuclides with detectable $\gamma$ - 

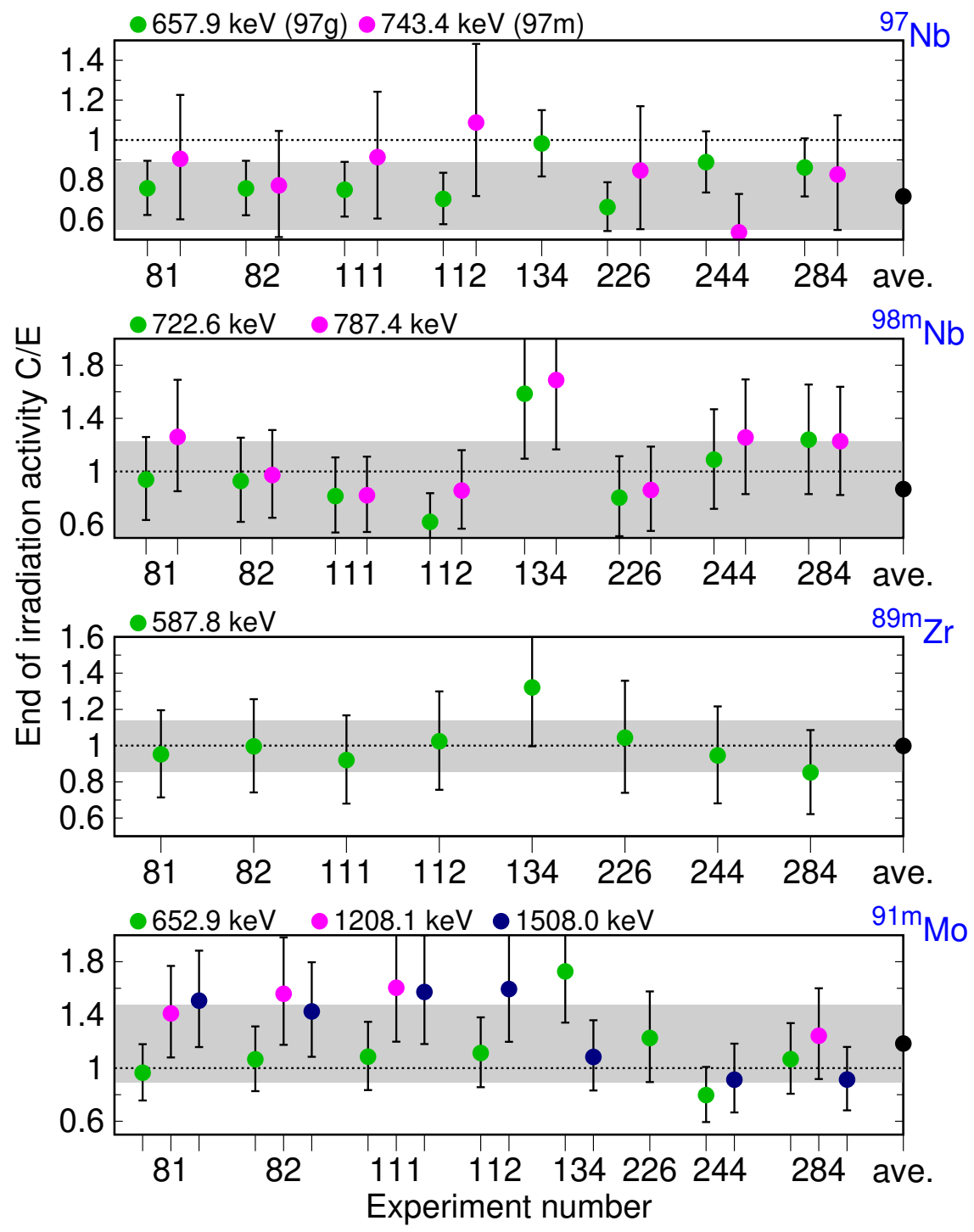

Figure 2: The complete set of $C / E$ values calculated for the ASP experiments involving Mo foils. The colour of a point indicates which $\gamma$-peak it is associated with (listed at the top of each pane). Points are grouped by experiment along the $x$-axis. Each pane corresponds to the peaks of a different radionuclide, except for the top pane, where the single measured peaks from each of ${ }^{97} \mathrm{Nb}$ and ${ }^{97 m} \mathrm{Nb}$ are shown together. The weighted-average $C / E$ value for all peaks in each pane is also plotted in black, and the grey band behind the data represents the standard deviation of the $C / E$ values from this average. See the main text for details.

emissions. Comparison to simulations of the experiment, performed by FISPACT-II [12] with the TENDL-2017 [22] library, revealed a good agreement $(C / E$ values near to one) despite the large degree of experimental uncertainty and statistical scatter.

While these tests help to improve confidence in some aspects of code predictions for Mo in a fusion environment, further validation is required, particularly for the production rates of the longlived radionuclides that dominate at long decay times under fusion reactor conditions. The short irradiation timescales associated with the experiments prevent detailed interrogation and testing 
of the production routes of long-lived radionuclides; further experiments, involving longer irradiation and measurement times and/or higher neutron fluences, are required. It may be possible to measure the cross sections of the relevant long-lived nuclides produced in Mo by irradiating larger samples at ASP for longer periods at lower fluxes (e.g. as parasitic experiments added to other campaigns) followed by longer $\gamma$-spectroscopy measurements in low-background conditions taken at intermittent intervals over the course of several months. Then there is a chance that weak, but relatively constant $\gamma$-signals from long-lived nuclides could be observed and used to evaluate cross sections. However, such measurements will have higher uncertainties due to the larger variation in conditions during the course of the experiments. None of the currently available devices have the required neutron fluxes, energies or experimental availability to fully meet the needs of fusion as it moves away from a research focus to a more engineering realisation phase with DEMO and the other near-commercial devices planned; a dedicated high-flux, high-availability $14 \mathrm{MeV}$ neutron source is urgently needed.

The validation efforts described here and planned for the future at UKAEA are intended to help to promote tailoring of Mo as a viable fusion option and to justify the ongoing industrial $R \& D$ into producing biased molybdenum at the necessary scale for fusion power plants.

\section{ACKNOWLEDGEMENTS}

This work was funded by the RCUK Energy Programme [grant number EP/T012250/1]. To obtain further information on the data and models underlying this paper please contact PublicationsManager@ukaea.uk.

\section{REFERENCES}

[1] J. Brooks, L. El-Guebaly, A. Hassanein, and T. Sizyuk. Nucl Fus, volume 55(4), p. 043002 (2015). https: //doi.org/10.1088/0029-5515/55/4/043002.

[2] M. R. Gilbert, L. W. Packer, J. Sublet, and R. A. Forrest. Nucl Sci Eng, volume 177(3), pp. $291-306$ (2014). https://doi.org/10.13182/NSE13-76.

[3] M. Rieth et al. J Nucl Mater, volume 432(1), pp. 482-500 (2013). https://doi.org/10.1016/j.jnucmat.2012.08. 018.

[4] Y. Ueda et al. Nucl Fus, volume 57(9), p. 092006 (2017). https://doi.org/10.1088/1741-4326/aa6b60.

[5] C. N. Taylor, Y. Yamauchi, M. Shimada, Y. Oya, and Y. Hatano. Fus Sci Tech, volume 71(4), pp. 491-495 (2017). https://doi.org/10.1080/15361055.2016.1273699.

[6] M. Gilbert, T. Eade, T. Rey, R. Vale, C. Bachmann, U. Fischer, and N. Taylor. Nuclear Fusion, volume 59(7), p. 076015 (2019). https://doi.org/10.1088/1741-4326/ab154e.

[7] A. Smirnov, A. Bonarev, G. Sulaberidze, V. Borisevich, G. Kulikov, and A. Shmelev. Physics Procedia, volume 72, pp. 126 - 131 (2015). https://doi.org/10.1016/j.phpro.2015.09.034.

[8] T. Noda, H. Suzuki, H. Araki, J. L. Lyman, and B. E. Newnam. J Nucl Mater, volume 307-311, pp. 715 - 718 (2002). https://doi.org/10.1016/S0022-3115(02)01265-5.

[9] L. Morgan, J. Shimwell, and M. Gilbert. "Isotopically enriched structural materials in nuclear devices." Fus EngDes, volume 90, pp. 79 - 87 (2015). https://doi.org/10.1016/j.fusengdes.2014.11.016.

[10] A. N. Shmelev and B. K. Kozhahmet. J Phys: Conf Series, volume 781, p. 012022 (2017). https://doi.org/10. 1088/1742-6596/781/1/012022. 
[11] F. Hernández and P. Pereslavtsev. Fus Eng Des, volume 137, pp. 243 - 256 (2018). https://doi.org/10.1016/j. fusengdes.2018.09.014.

[12] J. -Ch. Sublet, J. W. Eastwood, J. G. Morgan, M. R. Gilbert, M. Fleming, and W. Arter. Nucl Data Sheets, volume 139, pp. 77-137 (2017). http://dx.doi.org/10.1016/j.nds.2017.01.002.

[13] M. Fleming, J. -Ch. Sublet, M. R. Gilbert, A. Koning, and D. Rochman. EPJ Web Conf, volume 146, p. 02033 (2017). https://dx.doi.org/10.1051/epjconf/201714602033.

[14] R. D. Mosteller. "Bibliography of MCNP Verification and Validation: 1990 - 2003." Technical Report LA-UR03-9032, LANL (2003). Available as https://mcnp.lanl.gov/pdf_files/la-ur-03-9032.pdf.

[15] R. A. Forrest. Fus Eng Des, volume 81(18), pp. $2143-2156$ (2006). https://doi.org/10.1016/j.fusengdes.2006. 01.001.

[16] R. A. Forrest. Ene Procedia, volume 7, pp. 540-552 (2011). https://doi.org/10.1016/j.egypro.2011.06.075.

[17] L. W. Packer, M. Gilbert, S. Hughes, S. Lilley, R. Pampin, and J. -Ch. Sublet. Fus Eng Des, volume 87(5), pp. 662-666 (2012). https://doi.org/10.1016/j.fusengdes.2012.01.044.

[18] L. Packer, S. Hughes, M. Gilbert, S. Lilley, and R. Pampin. Fus Eng Des, volume 88(9), pp. 2617-2620 (2013). https://doi.org/10.1016/j.fusengdes.2013.05.108.

[19] M. R. Gilbert, L. W. Packer, and S. Lilley. Nucl Data Sheets, volume 119, pp. 401-403 (2014). https://doi.org/ 10.1016/j.nds.2014.08.112.

[20] T. Stainer et al. "14 MeV neutron irradiation data gamma spectroscopy analysis and validation automation." (2020). These proceedings.

[21] M. R. Gilbert and J. -Ch. Sublet. "Handbook of activation, transmutation, and radiation damage properties of the elements simulated using FISPACT-II \& TENDL-2015; Magnetic Fusion Plants." Technical Report CCFER(16)36, UKAEA (2016). Available from http://fispact.ukaea.uk.

[22] A. J. Koning and D. Rochman. “TENDL-2017.” Release Date: April 25, 2018. Available from https://tendl.web. psi.ch/tendl_2017/tendl2017.html. 